\title{
$X$-band photonic band-gap accelerator structure breakdown experiment
}

\author{
Roark A. Marsh* \\ Plasma Science and Fusion Center, Massachusetts Institute of Technology, Cambridge, Massachusetts 02139, USA, \\ and NIF \& Photon Science and Applications, Lawrence Livermore National Laboratory, Livermore, California 94550, USA \\ Michael A. Shapiro and Richard J. Temkin \\ Plasma Science and Fusion Center, Massachusetts Institute of Technology, Cambridge, Massachusetts 02139, USA
}

Valery A. Dolgashev, Lisa L. Laurent, James R. Lewandowski, A. Dian Yeremian, and Sami G. Tantawi SLAC National Accelerator Laboratory, Menlo Park, California 94025, USA

(Received 8 October 2010; published 4 February 2011)

\begin{abstract}
In order to understand the performance of photonic band-gap (PBG) structures under realistic high gradient, high power, high repetition rate operation, a PBG accelerator structure was designed and tested at $X$ band $(11.424 \mathrm{GHz})$. The structure consisted of a single test cell with matching cells before and after the structure. The design followed principles previously established in testing a series of conventional pillbox structures. The PBG structure was tested at an accelerating gradient of $65 \mathrm{MV} / \mathrm{m}$ yielding a breakdown rate of two breakdowns per hour at $60 \mathrm{~Hz}$. An accelerating gradient above $110 \mathrm{MV} / \mathrm{m}$ was demonstrated at a higher breakdown rate. Significant pulsed heating occurred on the surface of the inner rods of the PBG structure, with a temperature rise of $85 \mathrm{~K}$ estimated when operating in $100 \mathrm{~ns}$ pulses at a gradient of $100 \mathrm{MV} / \mathrm{m}$ and a surface magnetic field of $890 \mathrm{kA} / \mathrm{m}$. A temperature rise of up to $250 \mathrm{~K}$ was estimated for some shots. The iris surfaces, the location of peak electric field, surprisingly had no damage, but the inner rods, the location of the peak magnetic fields and a large temperature rise, had significant damage. Breakdown in accelerator structures is generally understood in terms of electric field effects. These PBG structure results highlight the unexpected role of magnetic fields in breakdown. The hypothesis is presented that the moderate level electric field on the inner rods, about $14 \mathrm{MV} / \mathrm{m}$, is enhanced at small tips and projections caused by pulsed heating, leading to breakdown. Future PBG structures should be built to minimize pulsed surface heating and temperature rise.
\end{abstract}

DOI: 10.1103/PhysRevSTAB.14.021301

PACS numbers: 29.20.Ej, 52.80.Pi, 52.80.Vp

\section{INTRODUCTION}

Photonic crystals or photonic band-gap (PBG) structures provide a groundbreaking starting point for advanced accelerator structure design. Photonic crystals are made up of an array of varying dielectric or metallic structures that affect frequency bands of electromagnetic waves passing through them, prohibiting all propagation within a band gap when it exists [1,2]. In application to accelerator structures, PBG structures have the ability to confine modes in a frequency band of interest, and damp modes of higher (or lower) frequencies. This allows PBG structures to be fabricated as a novel accelerator concept incorporating simultaneous damping of all higher order modes (HOMs). The use of a metallic PBG structure as an accelerator was first proposed based on a square metallic lattice in [3]. A structure with a triangular lattice has improved symmetry for accelerators and good agreement has been observed between network analyzer measurements and simulations [4]. A six cell traveling wave structure has also been built, based on this first design

*marsh19@1lnl.gov
[5]. This PBG structure demonstrated acceleration using a photonic structure for the first time [6]. HOMs in PBGs have been simulated, and PBG wakefields have also been measured [7-9].

Recent advances in the study of photonic structures for accelerator applications include research on hybrid structures consisting of metallic and dielectric components $[10,11]$; research on quasicrystals [12]; research on disordered photonic structures [13]; research on truncated, optimized photonic structures [14]; and theoretical research on wakefields [15].

The operation of PBG structures under high power and high repetition rate, especially with respect to breakdown performance has not been studied. To obtain first results on both electric and magnetic field effects in PBG structures at realistic operating conditions, a single cell standing wave structure has been designed and tested at SLAC, along the lines of other single cell standing wave structure tests [16-19]. SLAC has conducted extensive tests of conventional, pillbox structures at $11.424 \mathrm{GHz}$. The single PBG test cell was designed to create the highest electric and magnetic field in the test cell. The input and end cells were conventional pillbox cells, rather than photonic structures. 


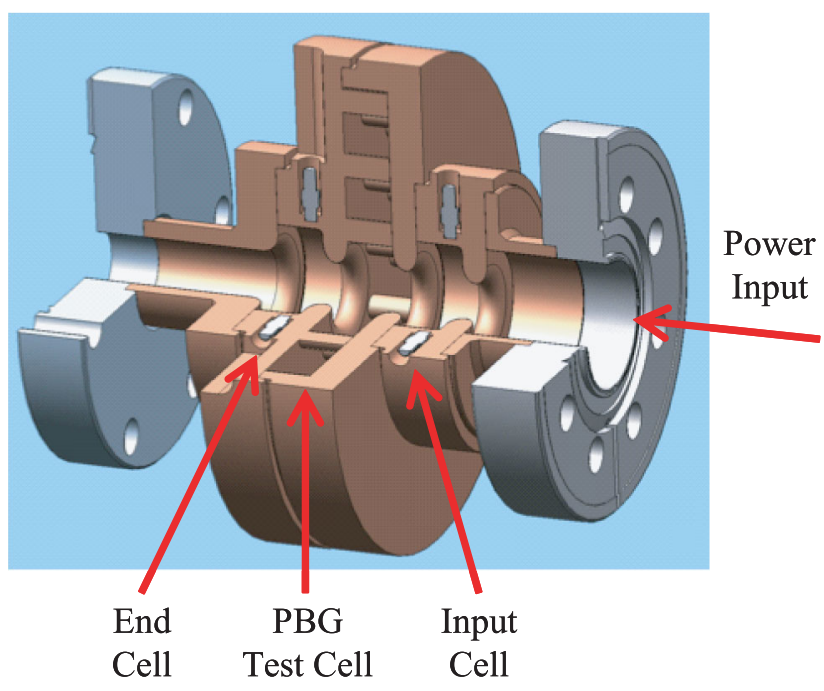

FIG. 1. Single cell standing wave PBG structure.

Power is injected into the structure using a $T M_{01}$ mode launcher [20]. These launchers had been previously built and successfully tested with conventional pillbox test structures. The PBG structure is shown in Fig. 1, with labels indicating the pillbox input and end cells, and the PBG test cell. Single cell testing has advantages over testing extended structures, e.g., the smaller structures require less power for testing, and are much easier to fabricate and tune. To date, more than 30 structures have been tested at SLAC, of varying iris aperture and geometry, different materials, and widely diverging geometries including choke mode structures [21] and this first PBG structure test.

\section{DESIGN OF THE PHOTONIC BAND-GAP (PBG) STRUCTURE}

The PBG test structure shown in Fig. 1 was designed using HFSS [22]. The structure was made to be as similar to standard pillbox structures as possible in order to compare with conventional structures. The specific pillbox cavity that was used as a starting point of the PBG design is designated as structure 1C-SW-A5.65-T4.6-Cu. It includes elliptical irises, which decrease the peak surface electric field to accelerating gradient ratio [23,24]. The elliptical cross-section irises improve the breakdown performance of the structure by raising the accelerating gradient achieved at a given breakdown rate. The iris geometry of the PBG structure, which is designated 1C-SW-A5.65-T4.6-PBG$\mathrm{Cu}$, was made identical to that of $1 \mathrm{C}-\mathrm{SW}-\mathrm{A} 5.65-\mathrm{T} 4.6-\mathrm{Cu}$, so that the PBG cell impact on structure performance could be isolated.

\section{A. PBG accelerator structure}

The PBG design required an operating mode with half field in the input and end cells and full field in the PBG cell, a mode frequency of $11.424 \mathrm{GHz}$, and near critical coupling into the entire structure. The PBG accelerator design has focused on designing a structure around a lattice that contains the desired accelerating mode and suppresses the higher order modes. Figure 2 shows a triangular lattice of cylindrical rods, with radii $\alpha$, and lattice spacing $\beta$. A defect is formed by removing a single, central rod from the lattice; a mode excited in proximity to the defect with a frequency within the band gap of the lattice will be confined to the defect, forming a high quality factor $(Q)$ resonator. In the triangular lattice of a PBG structure, there are two free parameters, $\alpha$ and $\beta$. However, the requirement to operate at a specific frequency $(11.424 \mathrm{GHz})$ reduces the design to one free parameter, which is taken as the ratio of rod radius to lattice spacing $(\alpha / \beta)$. PBG accelerator structures have been tested at an $\alpha / \beta$ ratio of 0.15 , and successfully confined a $T M_{01}$ mode [4-6]. An $X$-band scaling of this first structure, also with an $\alpha / \beta$ ratio of 0.15 , has also been tested via beam excitation [8].

Larger rods (increasing $\alpha / \beta$ ) improve the maximum surface electric field to maximum surface magnetic field ratio, which will lower the structure pulsed heating [25]. Using HFSS simulations, a positive variation in electric to magnetic peak surface field ratio is shown in Fig. 3; larger

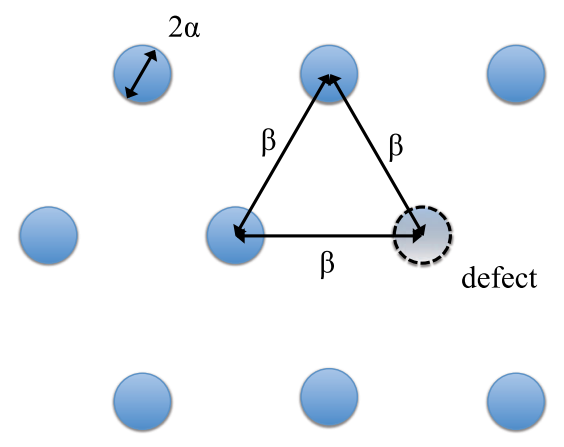

FIG. 2. Definition of $\alpha / \beta$ ratio.

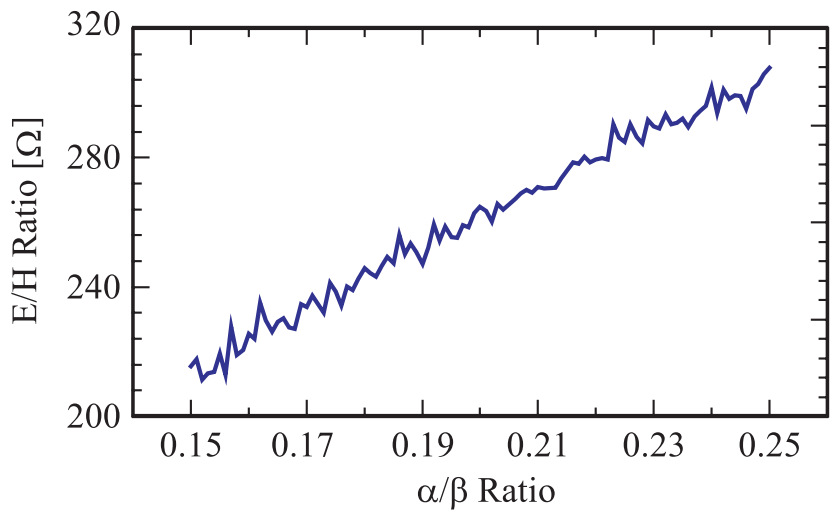

FIG. 3. Peak surface electric field to peak surface magnetic field ratio ( $\mathrm{E} / \mathrm{H}$ ratio) versus $\alpha / \beta$ ratio as calculated using HFSS. A linear increase in electric to magnetic surface field ratio is seen as $\alpha / \beta$ is raised. 


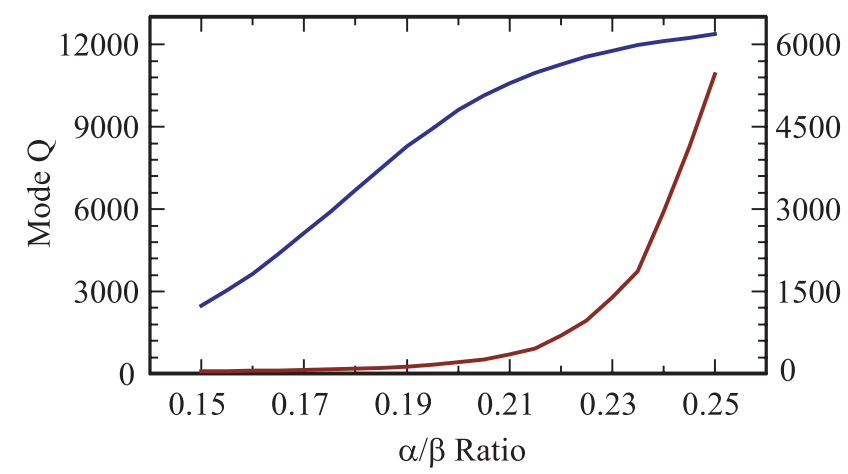

FIG. 4. $Q$ of fundamental mode (blue, left axis) and dipole mode (red, right axis) as a function of $\alpha / \beta$.

rods mean less magnetic field for a given surface electric field or gradient. The jaggedness of the line is a result and measure of the numerical resolution of the HFSS calculated fields.

Though larger rods mean an improvement in the peak surface magnetic field in the structure, which will mean lower pulsed heating, larger rods also confine PBG modes better. This applies not only to the fundamental operating mode of the structure, but also to HOMs. The fundamental $\left(T M_{01}\right)$ and dipole $\left(T M_{11}\right)$ mode $Q$ s are shown in Fig. 4 for
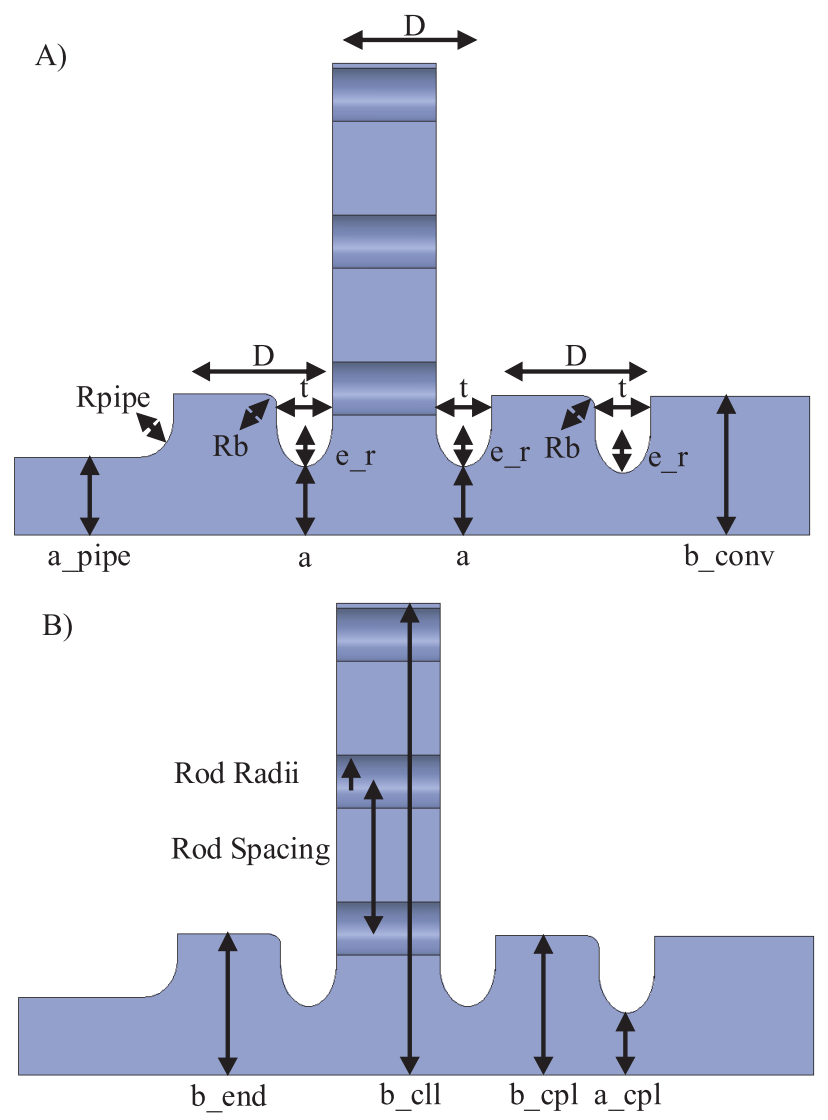

FIG. 5. Geometric parameters of PBG structure: (A) fixed parameters, (B) free tuning parameters, as shown in Tables I and II, respectively.
TABLE I. Fixed parameters for PBG structure, values for Fig. 5(a).

\begin{tabular}{lc}
\hline \hline \multicolumn{1}{c}{ Fixed parameters } \\
\hline Rpipe & $3 \mathrm{~mm}$ \\
Rb & $1 \mathrm{~mm}$ \\
t & $4.6 \mathrm{~mm}$ \\
e_r & $3.4 \mathrm{~mm}$ \\
D & $13.116 \mathrm{~mm}$ \\
a & $5.6475 \mathrm{~mm}$ \\
a_pipe & $6.35 \mathrm{~mm}$ \\
b_conv & $11.43 \mathrm{~mm}$ \\
\hline \hline
\end{tabular}

simulations including both Ohmic and diffractive losses [25]. The fundamental and dipole mode $Q$ s both increase with increasing $\alpha / \beta$, but the dipole mode $Q$ increases more rapidly. This is seen clearly in the ratio of fundamental to dipole mode $Q$ which shows a maximum at an $\alpha / \beta$ ratio of $\sim 0.17$. Increasing $\alpha / \beta$ will mean that, while pulse heating due to rf currents on the rods will be a smaller concern, the HOM damping attributes desired in a PBG structure will be compromised. An $\alpha / \beta$ ratio of 0.18 is an improvement in electric to magnetic peak surface field ratio performance over the previous 0.15 , and was chosen for this first single cell test.

An axisymmetric cutaway view of the PBG breakdown structure is shown in Fig. 5. The central test cell is constrained to have the iris geometry specified in Table I. For this given iris shape the cell is tuned so that it is the same as a single cell in a periodic structure with a $\pi$ phase advance per cell. The input and end pillbox coupling cells are then tuned to get an on axis electric field profile with half field in each coupling cell, and full field in the central PBG cell. The coupling aperture is then tuned to provide near critical coupling into the structure; for this design the structure was tuned to be slightly overcoupled. The final design properties are shown in Table II.

\section{B. Design results}

The results of the design process are indicated by the final tuning parameter values shown in Table II. Design properties and field results are from final HFSS driven mode solutions for an input power of $5.9 \mathrm{MW}$, which corresponds

TABLE II. Tuning parameters for PBG structure. Final design values for Fig. 5(b).

\begin{tabular}{lc}
\hline \hline \multicolumn{2}{c}{ Tuning parameters } \\
\hline b_end & $11.63 \mathrm{~mm}$ \\
b_cll & $38.87 \mathrm{~mm}$ \\
b_cpl & $11.47 \mathrm{~mm}$ \\
a_cpl & $5.13 \mathrm{~mm}$ \\
Rod radii & $2.176 \mathrm{~mm}$ \\
Rod spacing & $12.087 \mathrm{~mm}$ \\
\hline \hline
\end{tabular}


to an accelerating gradient of $100 \mathrm{MV} / \mathrm{m}$. A $30^{\circ}$ section of the full structure was used, corresponding to the irreducible geometric section of the triangular PBG lattice; all other components are cylindrically symmetric and insensitive to symmetry boundary conditions.

The normalized axial electric field is shown in Fig. 6: half field is seen in both the input and end cells, with peak field on axis in the PBG cell. Figure 7 shows calculated reflection $\left(S_{11}\right)$ as a function of frequency, showing a good match of $-24 \mathrm{~dB}$ at $11.4236 \mathrm{GHz}$. The structure is slightly overcoupled, as preferred.

Field plots are shown in Fig. 8 for the electric and magnetic fields as viewed along a radial cut of the structure through the nearest rod, for 5.9 MW input power (100 MV/m accelerating gradient). The electric field is strongest in the central cell, with peaks on axis and on the structure irises. The magnetic field is strongly peaked on the inner edge of the first row of rods. Top views of the electric and magnetic fields are shown in Fig. 9, for $5.9 \mathrm{MW}$ input power (100 MV/m accelerating gradient). The peak fields at the structure wall are listed in Table III.

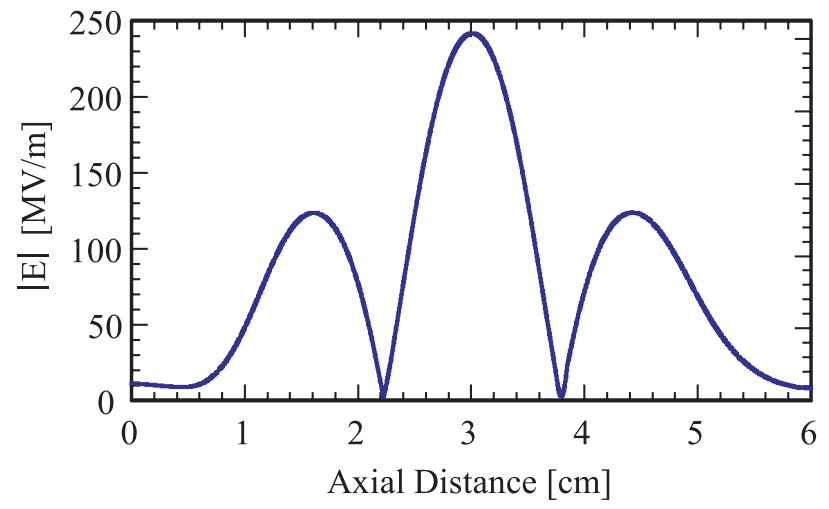

FIG. 6. Electric field relative magnitude on axis of PBG structure. Power is coupled in from the left; half field is seen in each coupling cell, relative to the central PBG cell.

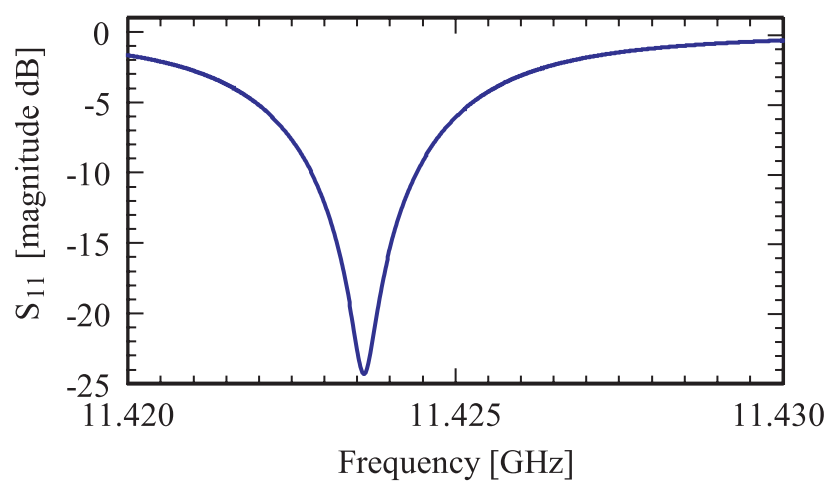

FIG. 7. Reflection as a function of frequency for PBG structure, as calculated.

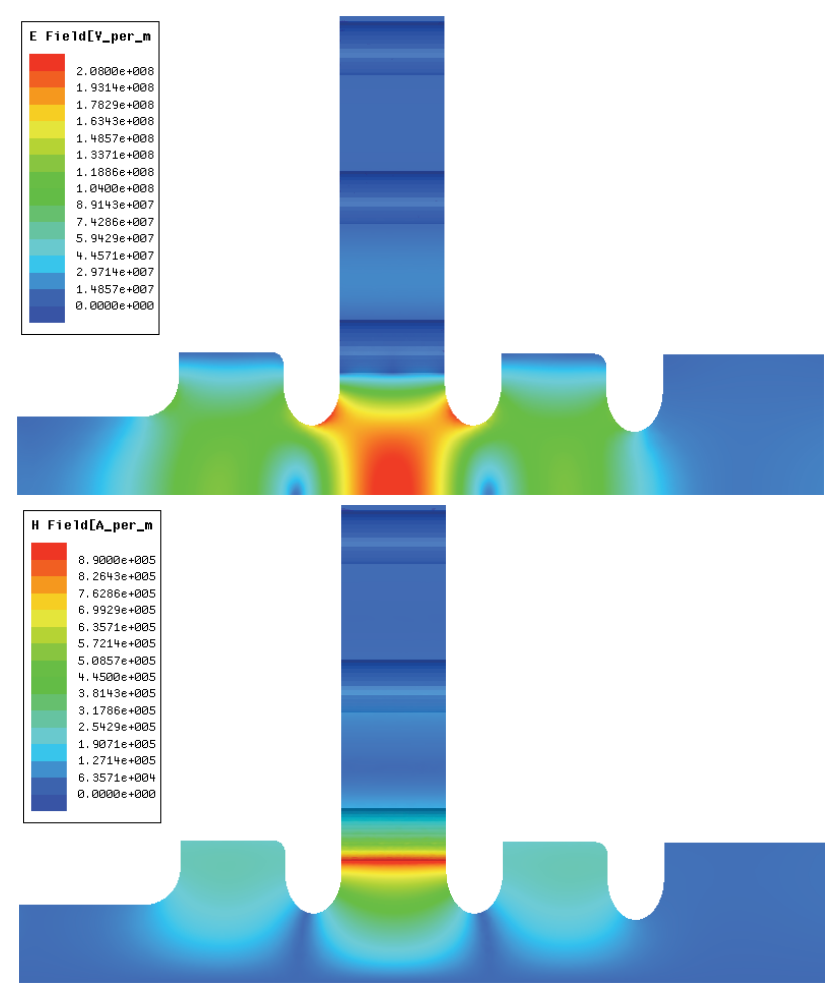

FIG. 8. Electric and magnetic field for PBG structure. Side views for 5.9 MW input power which corresponds to $100 \mathrm{MV} / \mathrm{m}$ accelerating gradient.

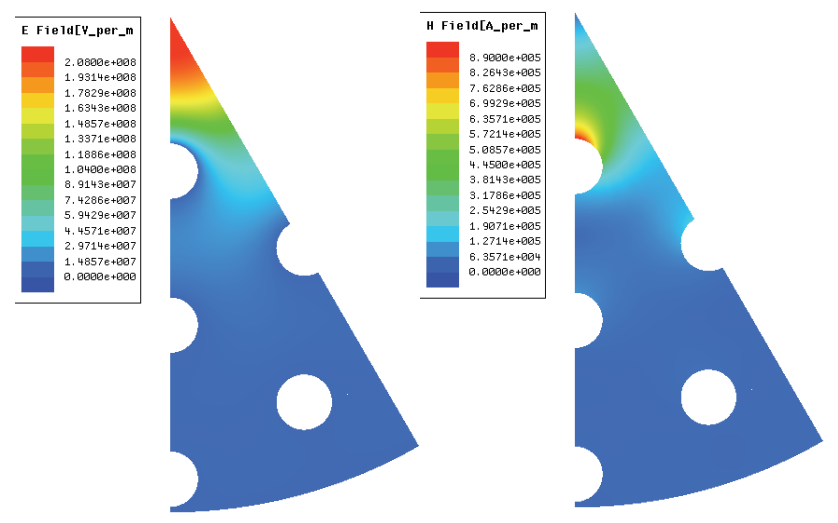

FIG. 9. Electric and magnetic field for PBG structure. Top views for 5.9 MW input power which corresponds to $100 \mathrm{MV} / \mathrm{m}$ accelerating gradient.

\section{HFSS analysis simulations \\ 1. $P B G$ simulations}

In the experiment, breakdown rates are measured as a function of input power levels to the structure. Breakdown rates are of interest not with regard to power levels, but with respect to the field properties (maximum surface electric and magnetic fields) of the accelerator structures. To convert from power level to field properties, HFSS simulations are used with an increased focus on field 
TABLE III. HFSS simulation field calibration numbers for PBG structure.

\begin{tabular}{lcc}
\hline \hline Structure & PBG & Pillbox \\
\hline Power & $5.9 \mathrm{MW}$ & $4.6 \mathrm{MW}$ \\
Gradient & $100 \mathrm{MV} / \mathrm{m}$ & $100 \mathrm{MV} / \mathrm{m}$ \\
Surface electric field maximum & $208 \mathrm{MV} / \mathrm{m}$ & $211 \mathrm{MV} / \mathrm{m}$ \\
Surface magnetic field maximum & $890 \mathrm{kA} / \mathrm{m}$ & $418 \mathrm{kA} / \mathrm{m}$ \\
Rod surface electric field & $14 \mathrm{MV} / \mathrm{m}$ & - \\
\hline \hline
\end{tabular}

convergence. Driven mode solutions in HFSS allow power levels to be calibrated with surface fields; a single calibration point suffices as the field scales as the square root of the power. The simulation for this calibration is run with as detailed a mesh as possible.

The accelerating gradient is calculated using the HFSS field calculator. Field convergence is a serious concern for such a critical field parameter, and this was ensured by steadily finer mesh operations. The suitability of this mesh was confirmed by calculating the gradient on a large number of slightly off axis lines. The gradient calculations were nearly constant, with small fluctuations as a result of field sampling.

The peak surface fields, both electric and magnetic, are also of interest for breakdown statistics. These are calculated using the eigenmode HFSS simulations and determining the maximum field value on the surface.

Cross calibration of driven and eigenmode HFSS simulations combine all of these field and power numbers so that the power coupled into the structure can be scaled into accelerating gradient, peak surface electric field, or peak surface magnetic field. A summary of the calibration parameters is given in Table III.

The electric field on the inner rod is of interest because that is the location where effects from both magnetic and electric fields interact. The peak electric field amplitude is much lower than on the structure iris. This can be compared by the values in Table III: for $100 \mathrm{MV} / \mathrm{m}$ gradient, the peak surface electric field on the iris is $208 \mathrm{MV} / \mathrm{m}$, and on the rod it is $14 \mathrm{MV} / \mathrm{m}$.

\section{Pillbox simulations}

This simulation process was repeated for the SLAC 1CSW-A5.65-T4.6-Cu baseline pillbox structure. These simulations were more straightforward than the PBG simulations because of the cylindrical symmetry of the pillbox structure; very fine meshes were possible with less reliance on meshing operations and sensitivity to field convergence. The calibration results are shown in Table III.

\section{Temperature rise calculation}

Comparison of the fields in the PBG and pillbox structures indicates design dictated similarities, along with some important differences. Comparing the maximum field values in Table III shows that the PBG structure has

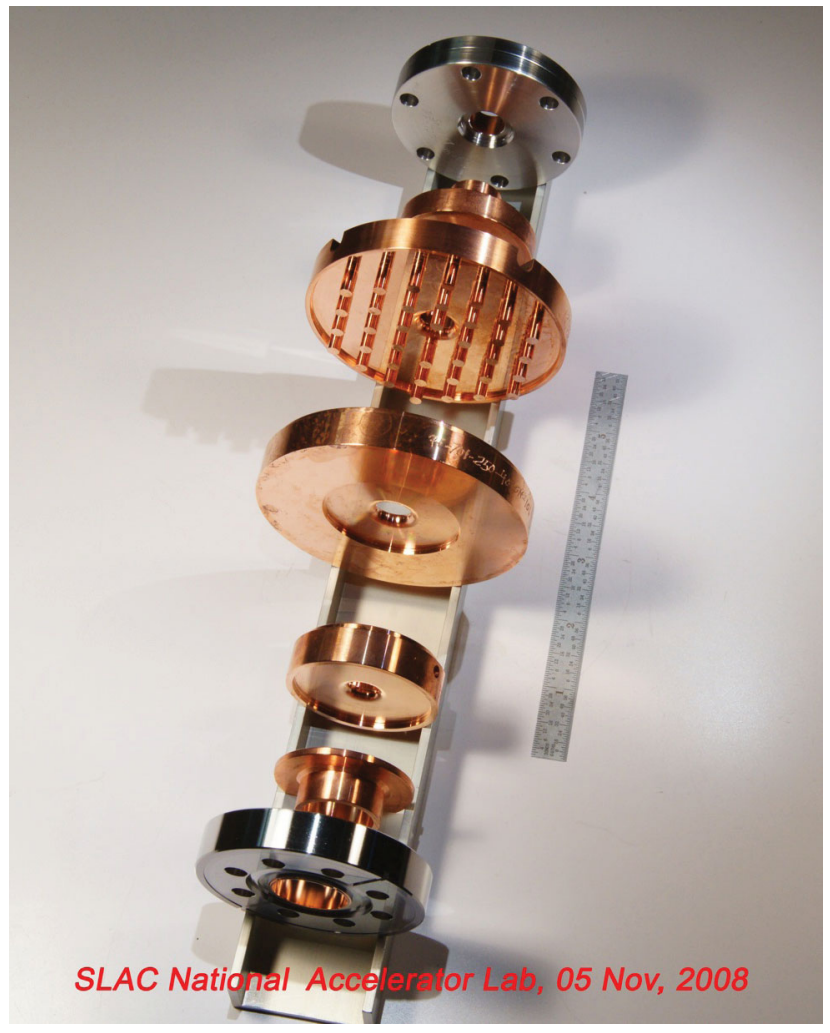

FIG. 10. PBG structure copper parts prior to brazing. A $152.4 \mathrm{~mm}$ ruler is located next to the structure to indicate the scale.

a higher peak surface magnetic field, so that for the same input power, a lower accelerating gradient is achieved. The increased power required for the PBG structure to reach the same accelerating gradient as the pillbox structure will not be a problem in breakdown testing because the power requirement is relatively low, and additional power is easily accommodated. Increased peak surface magnetic field, however, will result in higher pulsed heating on the structure.

The effect of magnetic field on predicted pulsed heating induced temperature rise can be described mathematically. For realistic pulse shapes, the exact pulsed heating temperature rise can be calculated using the general integral formulation shown in Eq. (1) [26]:

$$
\Delta T=\frac{1}{\rho c_{\epsilon} \sqrt{\pi \alpha_{d}}} \int_{0}^{t} \frac{1}{\sqrt{t-t^{\prime}}} \frac{1}{2} R_{S}\left|H\left(t^{\prime}\right)\right|^{2} d t^{\prime} .
$$

Equation (1) relates the temperature rise, $\Delta T$, to the time integral of the surface magnetic field as a function of time, $H\left(t^{\prime}\right)$, with the values for $\rho, c_{\epsilon}, \alpha_{d}$, and $R_{S}$ given in Table IV [26]. Equation (1) simplifies to Eq. (2) for a square pulse of magnitude $H_{\text {peak }}$ and duration $t_{P}$ [27]:

$$
\Delta T=430 \sqrt{t_{P}[\mu s]}\left|H_{\text {peak }}[M A / m]\right|^{2} K .
$$

In the PBG structure, for $100 \mathrm{MV} / \mathrm{m}$ gradient and a $100 \mathrm{~ns}$ square pulse a temperature rise of $85 \mathrm{~K}$ is estimated. 
TABLE IV. Material properties of oxygen-free high conductivity copper for insertion into Eq. (1).

\begin{tabular}{lcc}
\hline \hline$\rho$ & Density & $8.95 \times 10^{3} \mathrm{~kg} / \mathrm{m}^{3}$ \\
\hline$c_{\epsilon}$ & Specific heat at constant strain & $385 \mathrm{~J} / \mathrm{kg} \mathrm{K}$ \\
$\alpha_{d}$ & Linear thermal expansion coefficient & $1.65 \times 10^{-5} \mathrm{~K}^{-1}$ \\
$R_{S}$ & Surface resistance & $0.0279 \Omega($ at $11.424 \mathrm{GHz})$ \\
\hline \hline
\end{tabular}

The exact temperature rise in the structure depends on the varied pulse shape which is discussed further in Sec. IV.

\section{Fabrication}

The PBG cell was machined from a single block of oxygen-free high conductivity copper including one of the cell iris plates. The other iris was included in the facing cell piece, into which the PBG lattice rods were brazed. The machined parts, prior to copper brazing and flange welding are shown in Fig. 10.

\section{COLD TEST}

The single cell standing wave PBG structure, identified by SLAC code as 1C-SW-A5.65-T4.6-PBG-Cu, was matched with a $T M_{01}$ mode launcher, T52219-1, for all cold testing, and the same launcher remained connected for high power testing, and final cold testing. A vector network analyzer (VNA) was used to measure the complex reflection. A dry nitrogen flow was provided through a straight length of WR-90 waveguide to preserve structure cleanliness, and limit measurement fluctuations due to humidity. Temperature of the PBG structure was monitored by a sensor lead attached to its body.

The PBG structure is a one port device and so the nonresonant perturbation technique was adopted in which the reflection from the structure was measured in order to calculate the electric field profile $[28,29]$. This method involves the suspension of a small dielectric perturbation, or "bead," on a thin dielectric wire. A mounting allows the smooth draw of the bead through the structure close to on axis. The mounting was attached with three bolts, threaded through springs, so that they can be adjusted to center the bead. The wire used was Ashaway 10/0 black monofilament $2 \mathrm{lb}$ Trilene; the bead was made of a small drop of superglue.

\section{A. Bead pull measurements}

The cold test enables measurement of the cavity mode structure, frequency, and $Q$ values. The mode field profile is calculated by identifying a resonance in $S_{11}$ on the VNA and performing a calibration over a frequency range that fully encloses the resonance (i.e., a full $Q$ circle when viewing the Smith chart), moving the bead perturbation through the structure and measuring the real and imaginary reflection (on or slightly off resonance) as a function of bead position. Field profiles were captured for all resonances seen in the broad $S_{11}$ measurement shown in Fig. 11 .

Three modes were expected in the PBG structure itself: the 0 mode, $\pi / 2$ mode, and $\pi$ mode. Two coupler modes are also observed, formed by multiple peaks in the oversized waveguide connecting the $T M_{01}$ launcher and the PBG structure. This accounts for all the peaks observed in Fig. 11. Primary interest is in the matching of the $\pi$ mode on axis field profile to its design prediction, which is shown in Fig. 12; the overall agreement is very good.

\section{B. Coupling}

Once the modes have been identified, their coupling can be measured without the presence of the bead. The mode

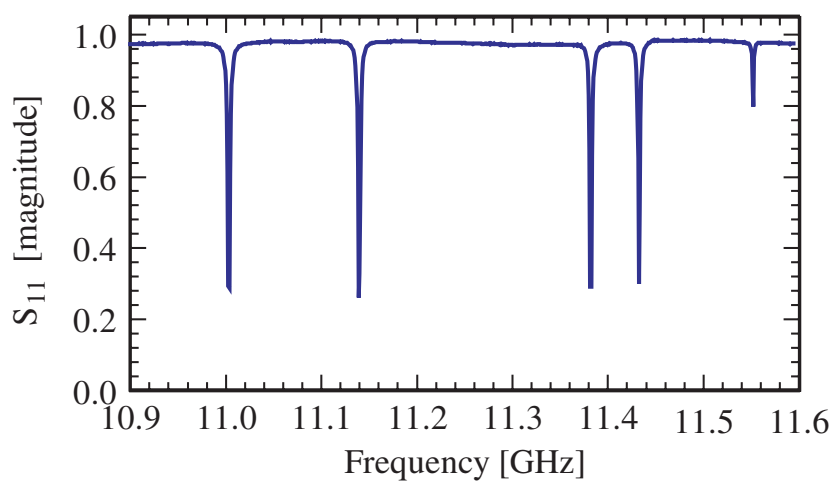

FIG. 11. Broad $S_{11}$ measurement showing all observed resonances of the PBG structure and mode launcher. The operating mode is the second from the right, at $11.43 \mathrm{GHz}$.

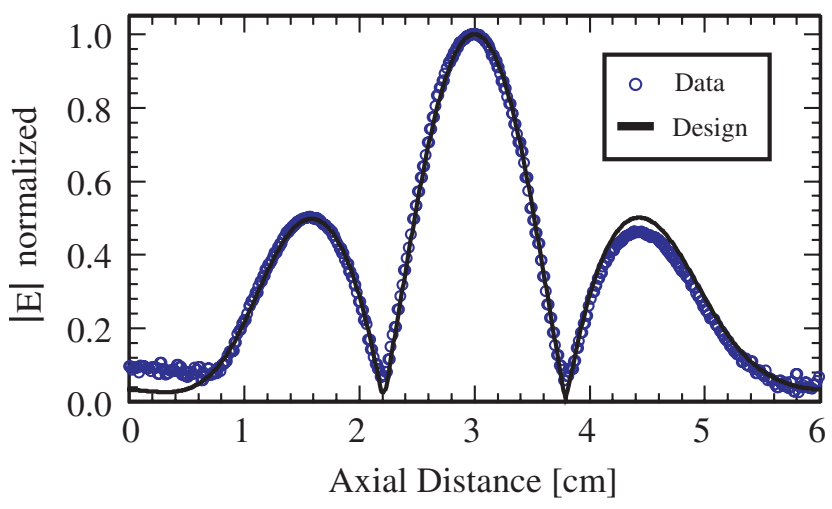

FIG. 12. Bead pull results for PBG standing wave structure. Very good agreement is seen between the cold test bead pull measurements and the design simulation. 
TABLE V. Table of measured $Q$ values for PBG structure modes.

\begin{tabular}{lcccr}
\hline \hline Mode & $\begin{array}{c}\text { Frequency } \\
{[\mathrm{GHz}]}\end{array}$ & $Q_{0}$ & $Q_{L}$ & $Q_{\text {ext }}$ \\
\hline 0 & 11.0036 & 8347 & 3390 & 5708 \\
$\pi / 2$ & 11.1397 & 9044 & 3796 & 6541 \\
Coupler & 11.3819 & 8299 & 4304 & 8941 \\
$\pi$ & 11.4323 & 7401 & 4695 & 12844 \\
Coupler & 11.5517 & 12746 & 11224 & 9395 \\
\hline \hline
\end{tabular}

frequency, coupling, and the mode $Q$ values can be determined [30]. Table $\mathrm{V}$ summarizes the mode $Q \mathrm{~s}$ : the unloaded $Q, Q_{0}$; the loaded $Q, Q_{L}$; and the external $Q$, $Q_{\text {ext }}$. The measured $\pi$-mode $Q_{0}$ of 7400 and the design simulation value of 7600 are in close agreement.

\section{EXPERIMENTAL SETUP}

The PBG breakdown structure was installed at the SLAC National Accelerator Laboratory on klystron test station \#4, which operates the $X$-band klystron XL4-6B and related microwave diagnostics. The tube is driven and controlled by computer, allowing pulse shaping and frequency tuning on a shot to shot basis. Two Agilent 8990 A peak power meters measure the fully calibrated klystron power, and the power being fed into the breakdown structure under test. The nominal power level is set and the klystron drive is changed to correct for drift in the klystron operating parameters. Filling of the standing wave structures is accomplished in a shaped manner; a high power level initially fills the structure, and then the power is lowered to maintain a constant level over the nominal power pulse length. Quoted power levels and pulse lengths correspond to this after filling steady state. The drive frequency is also tuned to maximally couple power into the structure by minimizing the reflected power; frequency tuning is necessary as the structure detunes with temperature by $190 \mathrm{kHz} / \mathrm{K}$. A $\log$ is kept for the test station, which provides information regarding operating conditions and the goals set for each operational run.

During the test, microwave diagnostics produce two sets of data: the peak power meter and scope traces. A typical peak power meter trace is shown in Fig. 13. Scope traces are fast scope measurements including: crystal detectors connected to directional couplers providing microwave measurements of structure forward and reverse power, and dark current measurement from current pickups, used as both forward and reverse Faraday cups (FCs). The scope traces are used as shot to shot diagnostics, and to monitor breakdown events. Breakdown events were recorded by observing an increase in reflected power from the structure, and the dark current Faraday cup measurements, which spike during breakdown shots. The total number of breakdowns in a given time span provides a breakdown rate,

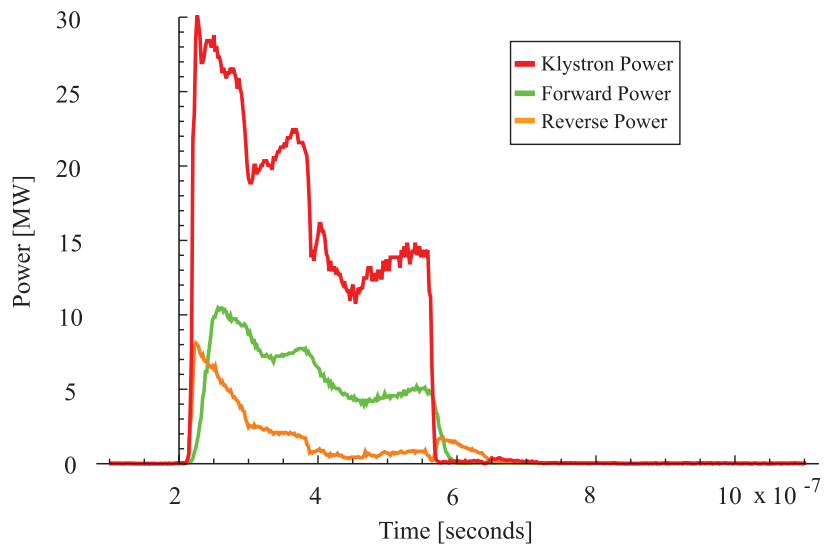

FIG. 13. Peak power meter trace for klystron signals: klystron output power, structure forward, and reverse power traces. After filling, the forward power into the structure was 5.0 MW for $170 \mathrm{~ns}$ for this shot.

quoted as number per hour at $60 \mathrm{~Hz}$ [19]. A typical scope trace is shown in Fig. 14, a typical scope trace during a breakdown shot is shown in Fig. 15; for both of these shots, the forward power into the structure (from the peak power meter) was 5.0 MW. The Faraday cup measurements were usually very low for the PBG structure, $\$ 2-3 \mathrm{~mA}$. During breakdown shots, however, Faraday cup measurements of $>45 \mathrm{~mA}$ were observed. The ringing visible on the saturated Faraday cup signals is from a small impedance mismatch at the scope input because the termination was optimized for small signals.

\section{Data}

Filtering useful shots for analysis is important for both the analysis process itself, and data storage. The peak power meter logs data continuously, storing shots every two seconds. The scope traces have a much higher volume, as they are taken continuously, every shot, while operating at a repetition rate of $60 \mathrm{~Hz}$. Rather than storing all shots,

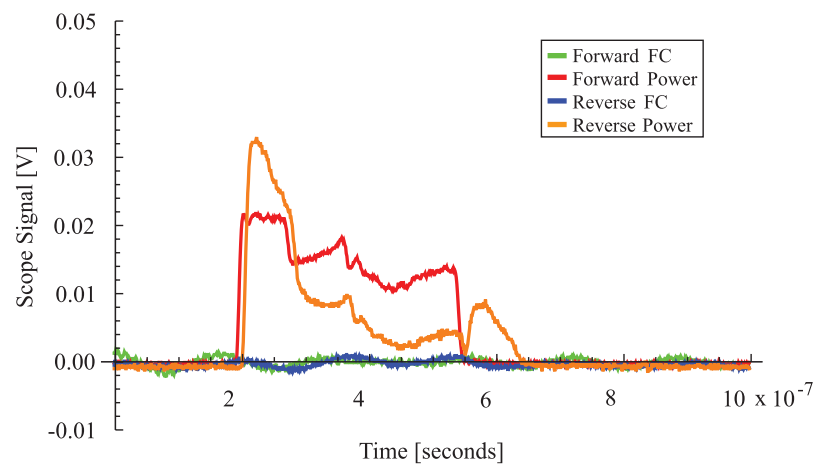

FIG. 14. Breakdown structure scope traces (uncalibrated) for nonbreakdown event. Forward and reverse power in structure, forward and reverse Faraday cup signals. After filling, the forward power into the structure (from the peak power meter) was 5.0 MW for $170 \mathrm{~ns}$ for this shot. 


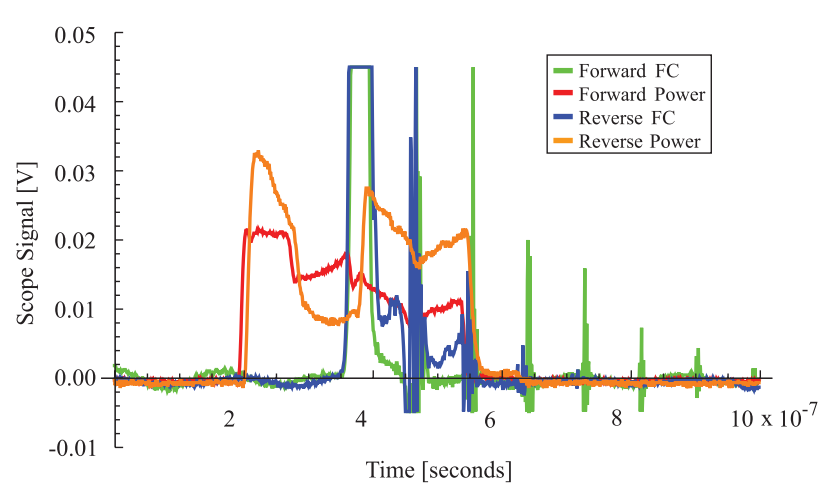

FIG. 15. Breakdown structure scope traces (uncalibrated) for breakdown event. Forward and reverse power in structure, forward and reverse Faraday cup signals. After filling, the forward power into the structure (from the peak power meter) was 5.0 MW for $170 \mathrm{~ns}$ for this shot.

scope traces are stored for every breakdown event, as well as the shot immediately preceding each breakdown event for direct comparison of operating parameters.

\section{DATA ANALYSIS}

The peak power meter and scope traces provide the bulk of information concerning the structure performance, but must be processed to provide useful structure information. Breakdown data is derived from the scope traces. Individual breakdown data sets were made up from all data gathered sequentially at a given power level and pulse length, as determined by the scope traces. Precise power levels and pulse length information are derived from peak power meter data with overlapping temporal coverage of each breakdown data set. These measured power levels are then scaled into structure field information by cross calibration with HFSS simulations. The accuracy of the calibrating simulations is confirmed by close agreement with structure cold test data. MATHEMATICA was used to handle the binary data sets, and individual programs were written to handle the individual tasks associated with the analysis [31].

\section{Data processing}

Scope traces contain both breakdown pulses, e.g., Fig. 15, and the pulses immediately prior, e.g., Fig. 14. Breakdown pulses are characterized by an increased power reflection prior to the termination of the forward power, and a dramatic increase in the dark current measured by the Faraday cups. For data processing purposes, an off-scale Faraday cup measurement of the dark current was used and verified as indicative of breakdown events. The scope traces provide breakdown data sets with time information, so that the total number of breakdowns in a given time span provides a breakdown rate, quoted after [19] as number per hour at $60 \mathrm{~Hz}$.

The time between breakdowns can be calculated directly from the data. The distribution of these points is given in

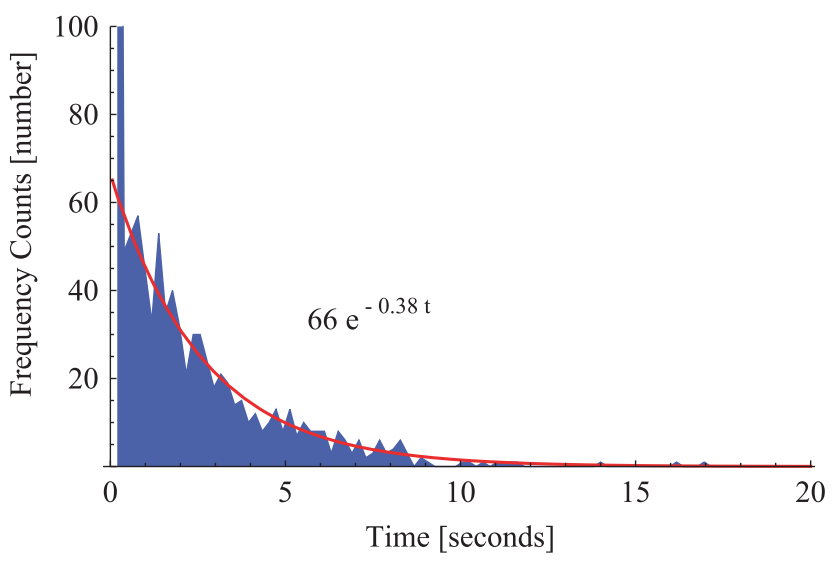

FIG. 16. Histogram of data taken at an accelerating gradient of $114 \mathrm{MV} / \mathrm{m}$ for $150 \mathrm{~ns}$ pulse length. Data is truncated to show detail; first bin height is actually 369. Total number of breakdowns is 1139. Exponential fit to data is made excluding the first bin, and yields $66 e^{-0.38 t}$.

the histogram of Fig. 16 for the data set corresponding to an accelerating gradient of $114 \mathrm{MV} / \mathrm{m}$ at $150 \mathrm{~ns}$ pulse length. A large number of breakdown events are followed immediately by a subsequent breakdown; a phenomenon that is usually observed in rf processing [32], and quantified in DC breakdown studies [33], but is readily quantifiable with the large collection of data resulting from this testing process. The percentage of immediate breakdown events ranges across all data sets from $\sim 25 \%-65 \%$. Excluding immediate breakdowns, the shape of the distribution is roughly exponential, and can be fit as shown in Fig. 16 for the $114 \mathrm{MV} / \mathrm{m} 150 \mathrm{~ns}$ data set.

Once breakdown data sets have been generated and a breakdown rate for the data set has been calculated, a power level is time correlated to the rate. The time indexing of the breakdown events gives a time span over which the relevant data was taken. The peak power meter data for this time span is used to calculate a pulse length and nominal power level. The calculated power level and pulse length are confirmed with the operator log.

Both the baseline pillbox structure, 1C-SW-A5.65-T4.6$\mathrm{Cu}$, and the PBG structure, 1C-SW-A5.65-T4.6-PBG-Cu, were analyzed with the same algorithms to provide as close a comparison as possible.

\section{EXPERIMENTAL RESULTS}

For the PBG structure, breakdown rates were observed for 150, 170, 300, 360, and 600 ns pulse lengths. Pillbox data existed for all of these pulse lengths, so that comparisons could be made between the performance of the two structures. Comparison of PBG and pillbox breakdown rates is shown as a function of peak surface electric field in Fig. 17 for 170 ns pulse length. For the same pulse shape, the breakdown rate in the PBG structure was higher than in the pillbox structure. This can be alternatively phrased that, 


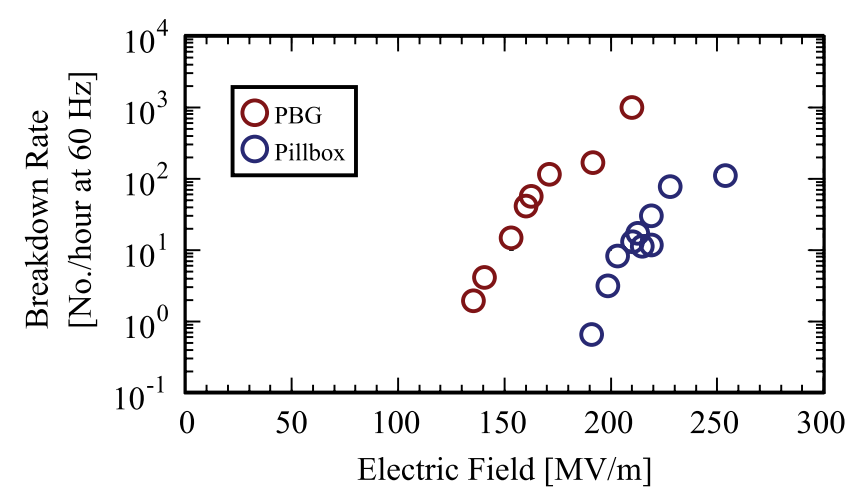

FIG. 17. PBG and pillbox breakdown rate vs maximum surface electric field for $170 \mathrm{~ns}$ pulse length.

for a given breakdown rate, the accelerating gradient or peak surface electric field in the PBG structure was lower than that in the pillbox structure.

Breakdown phenomena are generally understood in terms of maximum surface electric field. This is not what is seen in Fig. 17, as the performance of the two structures is not identical when compared in terms of peak surface electric field. Both the PBG and the pillbox structure have a surface field of $\sim 210 \mathrm{MV} / \mathrm{m}$ for a $100 \mathrm{MV} / \mathrm{m}$ accelerating gradient. This would predict similar breakdown performance for the structures.

Comparison of PBG and pillbox breakdown rates is shown as a function of peak surface magnetic field in Fig. 18 for $170 \mathrm{~ns}$ pulse length. The peak magnetic field is much higher in the PBG structure. Testing a structure with a higher ratio of peak surface magnetic field to peak electric field is very interesting because previous structures have similar ratios [16-19]. In [18] breakdown rate plots indicated that across many different structures, breakdown rate data seemed to collapse when plotted on a common pulsed heating axis. In [19] this same effect is observed when data is plotted with respect to peak surface magnetic field. The similarity of the structures with respect to their peak magnetic field was a strong motivator for testing a PBG structure with a much higher ratio of peak surface magnetic field to peak surface electric field.

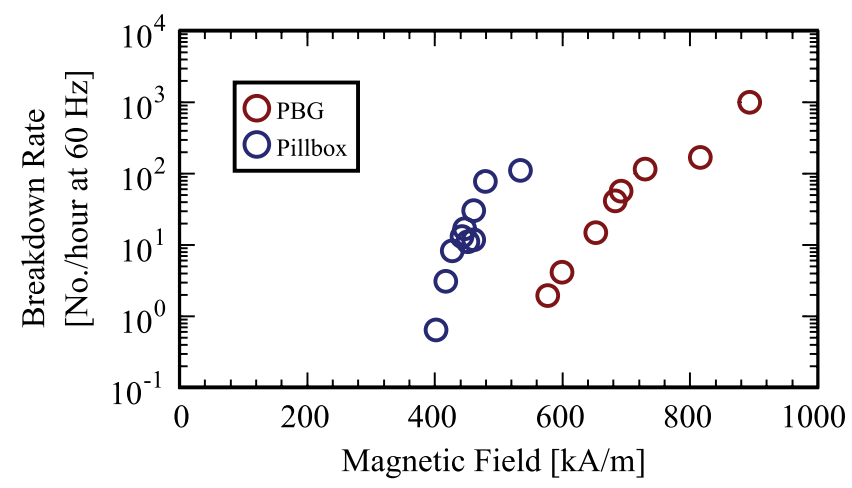

FIG. 18. PBG and pillbox breakdown rate vs maximum surface magnetic field for $170 \mathrm{~ns}$ pulse length.

\section{AUTOPSY}

After high power testing, nonresonant reflection measurements were repeated, and a decrease in $Q_{0}$ of the operating mode was observed from 4700 to 4200 . The structure was examined using a borescope. In borescope images, the iris appeared undamaged, and extensive damage was observed on the inner row of rods. The PBG structure was then cut in half, so that no inner rod was intercepted. Scanning electron microscope (SEM) micrographs were taken, and examples are shown in Figs. 19 and 20 of the PBG cell iris and an inner rod, respectively. Figure 19 shows a very smooth surface, indicating no visible damage to the iris. This is unusual; pillbox structures tested at high power and breakdown rate typically show damage at the iris. Figure 20 shows significant surface damage on an inner PBG rod, with the center of the rod, where the magnetic field is a maximum, showing the greatest damage. A detailed micrograph is shown in
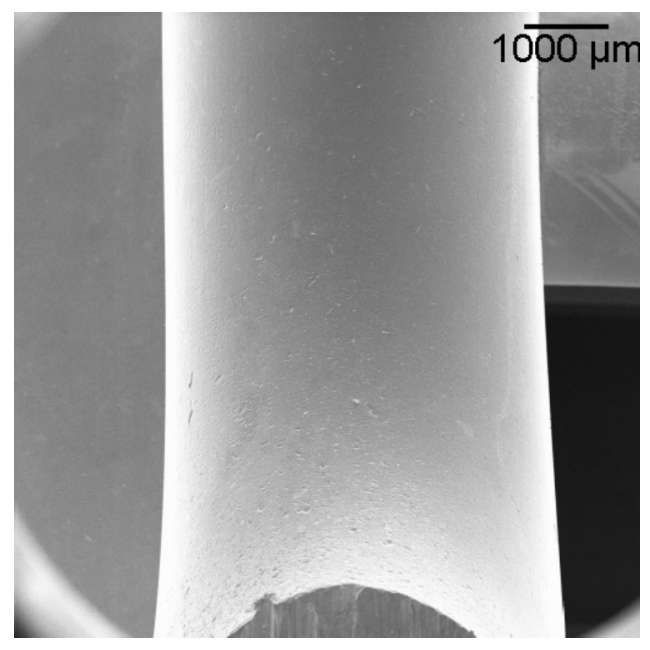

FIG. 19. SEM micrograph of PBG iris.

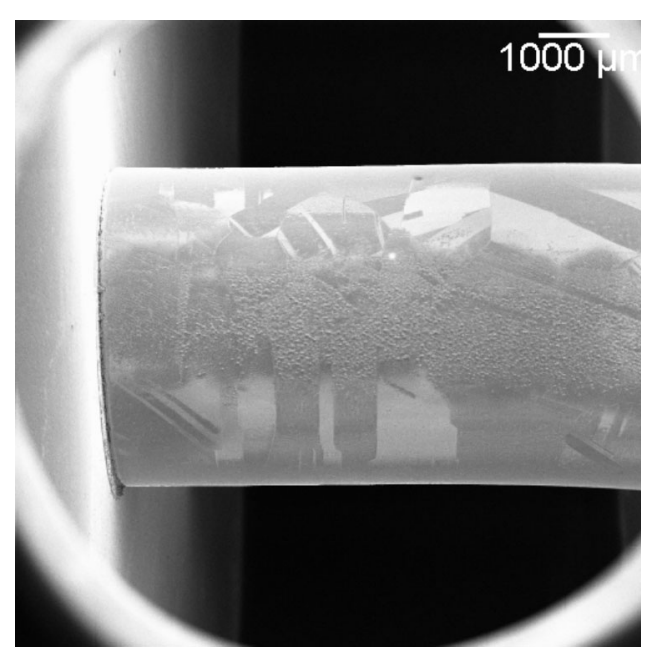

FIG. 20. SEM micrograph of inner rod. 


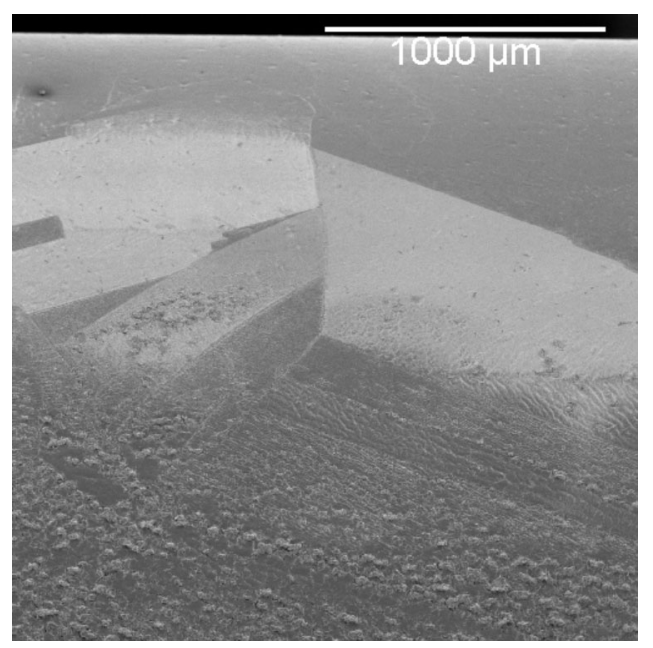

FIG. 21. SEM micrograph showing detail of inner rod pulsed heating damage.

Fig. 21; grain boundaries are visible, with increased surface roughness at the grain boundaries, and at the location of the peak magnetic field, near the center of the rod. The PBG structure experienced very high pulsed heating, with peak excursions to $>250 \mathrm{~K}$, and an average of $170 \mathrm{~K}$ for $35 \times 10^{6}$ shots, as calculated from the full data sets and Eq. (1). This level of pulsed heating temperature rise is responsible for the damage observed on the inner rod surfaces.

\section{DISCUSSION}

The PBG structure breakdown testing represents very exciting first results on high gradient damped structures. Breakdown results were compared with a pillbox structure for 150, 170, 300, 360, and $600 \mathrm{~ns}$ pulse lengths. For all data sets, the PBG structure had higher breakdown rates for comparable accelerating gradient, or peak surface electric field. The comparison shown in Figs. 17 and 18 for 170 ns pulse length is representative of the results at the other pulse lengths. The PBG structure was tested under the same conditions as all other structures [16-19]. If the performance of the single cell breakdown structures depended only on the iris geometry, or the peak surface electric field, then the performance of the PBG structure should have been identical to that of a similar pillbox, which it was not. The significant discrepancy between pillbox and PBG performance points very strongly to fundamental differences in the breakdown process in the two structures.

The PBG structure had much higher peak surface magnetic fields than a pillbox structure with similar iris geometry for the same gradient. These high magnetic fields produced large pulsed heating in the structure surface, and damage as probed by SEM. As commonly believed, pulsed heating is a disparate phenomenon, and does not drive breakdown processes. Pulsed heating is a physical limit on accelerating structure lifetime, requiring them to be designed with modest pulsed heating temperature rises of $<150 \mathrm{~K}[26,34]$. Lower pulsed heating limitations have also been advocated, requiring $<50 \mathrm{~K}$ to avoid observable surface damage. The performance of PBG structures should be on par with what has been achieved in pillbox structures, specifically the structure to which the single cell PBG structure has been compared in Figs. 17 and 18. The disparity in breakdown performance points to new physics, or new overlap in the physical processes involved in structure breakdown and pulsed heating, limits that are understood to be dominated by electric and magnetic fields, respectively.

One hypothesis for the PBG performance involves pulsed heating damage creating breakdown sites. Pulsed heating damage on the inner rods is observed over the peak magnetic field region, which is the high field facing surface of the inner row of rods; for $100 \mathrm{MV} / \mathrm{m}$ gradient, a peak magnetic field of $890 \mathrm{kA} / \mathrm{m}$ is present over this region. This high magnetic field results in pulsed heating temperature rises, in accordance with Eq. (1). The heating of the surface will cause cracking and an increase in the rod surface roughness. The increased surface roughness seen on the surface in Figs. 20 and 21 leads to a local concentration of the electric field on the roughened surface. The enhanced electric field may allow the surface electric field at the rods, which is only $14 \mathrm{MV} / \mathrm{m}$ at $100 \mathrm{MV} / \mathrm{m}$ accelerating gradient, to initiate breakdown at the rod. The combined effects of both high peak surface electric fields and high peak surface magnetic fields are responsible for structure performance [27].

Future linear accelerators will use damped structures to reach very high beam quality and luminosity. In the end it will be the performance of damped structures that will need to meet a breakdown rate specification, which requires further breakdown testing of damped structures. Further design work and experimental results are required to increase the accelerating gradient at which high breakdown rates result. Improved PBG structures have been designed, with decreased peak pulsed heating, and will be tested in an effort to both confirm these results and improve the high gradient performance of PBG structures [25].

\section{ACKNOWLEDGMENTS}

This work was supported by the Department of Energy High Energy Physics, under Contract No. DE-FG0291ER40648. The authors gratefully acknowledge useful discussions with Brian Munroe, Evgenya Smirnova, and Jake Haimson. R. A. Marsh would especially like to thank the MEGa-Ray team at $L L N L$ and Director George Miller for providing support to complete this paper.

[1] E. Yablonovitch, Phys. Rev. Lett. 58, 2059 (1987).

[2] S. John, Phys. Rev. Lett. 58, 2486 (1987). 
[3] D. R. Smith, S. Schultz, N. Kroll, M. Sigalas, K. M. Ho, and C. M. Soukoulis, Appl. Phys. Lett. 65, 645 (1994).

[4] M. A. Shapiro, W. J. Brown, I. Mastovsky, J. R. Sirigiri, and R. J. Temkin, Phys. Rev. ST Accel. Beams 4, 042001 (2001).

[5] E. I. Smirnova, I. Mastovsky, M. A. Shapiro, R. J. Temkin, L. M. Earley, and R. L. Edwards, Phys. Rev. ST Accel. Beams 8, 091302 (2005).

[6] E. I. Smirnova, A. S. Kesar, I. Mastovsky, M. A. Shapiro, and R. J. Temkin, Phys. Rev. Lett. 95, 074801 (2005).

[7] R. A. Marsh, M. A. Shapiro, R. J. Temkin, and E.I. Smirnova, in Proceedings of the 2007 Particle Accelerator Conference, Albuquerque, New Mexico (IEEE, Albuquerque, New Mexico, 2007), pp. 3002-3004.

[8] C. Jing, F. Gao, S. Antipov, Z. Yusof, M. Conde, J. G. Power, P. Xu, S. Zheng, H. Chen, C. Tang, and W. Gai, Phys. Rev. ST Accel. Beams 12, 121302 (2009).

[9] R. A. Marsh, M. A. Shapiro, R. J. Temkin, E. I. Smirnova, and J. F. DeFord, Nucl. Instrum. Methods Phys. Res., Sect. A 618, 16 (2010).

[10] M. R. Masullo, A. Andreone, E. Di Gennaro, S. Albanese, F. Francomacaro, M. Panniello, V. G. Vaccaro, and G. Lamura, Microw. Opt. Technol. Lett. 48, 2486 (2006).

[11] E. Di Gennaro, C. Zannini, S. Savo, A. Andreone, M. R. Masullo, G. Castaldi, I. Gallina, and V. Galdi, New J. Phys. 11, 113022 (2009).

[12] E. Di Gennaro, S. Savo, A. Andreone, V. Galdi, G. Castaldi, V. Pierro, and M. Rosaria Masullo, Appl. Phys. Lett. 93, 164102 (2008).

[13] C. J. Matthews and R. Seviour, Appl. Phys. B 94, 381 (2009).

[14] C. A. Bauer, G. R. Werner, and J. R. Cary, J. Appl. Phys. 104, 053107 (2008).

[15] G. R. Werner, C. A. Bauer, and J. R. Cary, Phys. Rev. ST Accel. Beams 12, 071301 (2009).

[16] V. A. Dolgashev, S. G. Tantawi, C. D. Nantista, Y. Higashi, and T. Higo, in Proceedings of the 2004 Linear Accelerator Conference, Lübeck, Germany, 2004 (DESY, Hamburg, 2004), pp. 766-768.

[17] V. A. Dolgashev, S. G. Tantawi, C. D. Nantista, Y. Higashi, and T. Higo, in Proceedings of the 21st Particle Accelerator Conference, Knoxville, 2005 (IEEE, Piscataway, NJ, 2005), pp. 595-599.
[18] V. A. Dolgashev, S. G. Tantawi, C. D. Nantista, Y. Higashi, and T. Higo, in Proceedings of the 2007 Particle Accelerator Conference, Albuquerque, New Mexico (Ref. [7]), pp. 2430-2432.

[19] V. A. Dolgashev, S. G. Tantawi, Y. Higashi, and T. Higo, in Proceedings of the 11th European Particle Accelerator Conference, Genoa, 2008 (EPS-AG, Genoa, Italy, 2008), pp. 742-744.

[20] C. Nantista, S. Tantawi, and V. Dolgashev, Phys. Rev. ST Accel. Beams 7, 072001 (2004).

[21] T. Shintake, Jpn. J. Appl. Phys. 31, L1567 (1992).

[22] High Frequency Structure Simulator, Ansoft Corporation, http://www.hfss.com.

[23] B. Spataro (private communication).

[24] O. Nezhevenko, D. Myakishev, V. Tarnetsky, and V. Yakovlev, in Proceedings of the Particle Accelerator Conference, Dallas, TX, 1995 (IEEE, New York, 1995), pp. 1076-1078.

[25] R. A. Marsh, B. J. Munroe, M. A. Shapiro, and R. J. Temkin, in Proceedings of the 23rd Particle Accelerator Conference, Vancouver, Canada, 2009 (IEEE, Piscataway, NJ, 2009), WE6RFP081.

[26] D. P. Pritzkau and R. H. Siemann, Phys. Rev. ST Accel. Beams 5, 112002 (2002).

[27] V. A. Dolgashev, in Proceedings of the 20th Particle Accelerator Conference, Portland, OR, 2003 (IEEE, New York, 2003), pp. 1267-1269.

[28] L. C. Maier Jr. and J.C. Slater, J. Appl. Phys. 23, 68 (1952).

[29] C. W. Steele, IEEE Trans. Microwave Theory Tech. 14, 70 (1966).

[30] David M. Pozar, Microwave Engineering (Wiley, New York, 2005), 3rd ed.

[31] MATHEMATICA 7, Wolfram Research, http://www .wolfram.com.

[32] C. Adolphsen, W. Baumgartner, K. Jobe, F. Le Pimpec, R. Loewen, D. McCormick, M. Ross, T. Smith, J. W. Wang, and T. Higo, in Proceedings of the 19th Particle Accelerator Conference, Chicago, Illinois, 2001 (IEEE, Piscataway, NJ, 2001), pp. 478-480.

[33] A. Descoeudres, Y. Levinsen, S. Calatroni, M. Taborelli, and W. Wuensch, Phys. Rev. ST Accel. Beams 12, 092001 (2009).

[34] O. A. Nezhevenko, in Proceedings of the 23rd Particle Accelerator Conference, Vancouver, Canada, 2009 (Ref. [25]), pp. 3013-3014. 